\title{
FAMILIES OF MUTUALLY COMPLEMENTARY TOPOLOGIES
}

\author{
B. A. ANDERSON ${ }^{1}$
}

ABSTRACT. Several lattices of topologies on an infinite set are considered and bounds are given for the sup of the set of cardinals $d$ such that there is a family of $d$ mutually complementary topologies. Large classes of $\boldsymbol{\aleph}_{\boldsymbol{\sigma}}$-topologies are shown to have $\boldsymbol{\aleph}_{\boldsymbol{0}}$-complements, and an example is given to prove that complementation is not, in general, a very selective topological operation.

1. Introduction. An example was given in [1] of three mutually $T_{1}$-complementary topologies on a denumerable set and the question was raised as to how many mutually $T_{1}$-complementary topologies an infinite set can carry. In [2] it was shown that every infinite set carries a collection of three mutually $T_{1}$-complementary topologies. If $L$ is a nonempty complete lattice, let us define the complementary width of $L$ (see [3, p. 98]), $w^{*}(L)$, to be the sup of the set of cardinals $d$ such that $L$ has a family of $d$ mutually complementary elements. One result of this paper is that if $X$ is an infinite set and $L$ is one of several important lattices of topologies on $X$, then $|X| \leqq w^{*}(L)$ $\leqq 2^{|\boldsymbol{X}|}$. Schnare [8] has considered the problem of determining the cardinality of the set of complements for any topology in the lattice of all topologies on a set.

It is known [10] that the lattice of $T_{1}$ topologies on an infinite set is not complemented. Recently several papers [1], [2], [10], [11] have dealt with the question of what $T_{1}$ topologies have $T_{1}$-complements. Some of the results of [2] can be carried over to another noncomplemented lattice of topologies; namely, the lattice of $\boldsymbol{\aleph}_{\mathbf{0}}$-topologies. This is done by showing that the complements exhibited in [2] are actually Fréchet topologies.

Lastly, an example is given to show how topologically undiscerning complementation is. It turns out that one topology can be complementary to topologies whose local properties of most points are completely arbitrary.

Received by the editors August 7, 1970.

AMS 1969 subject classifications. Primary 5240; Secondary 0415, 0635.

Key words and phrases. Topologies, principal topologies, $T_{1}$ topologies, $\boldsymbol{\aleph}_{\boldsymbol{0}}$-topologies, lattice complementation.

1 This research was supported by an Arizona State University Summer Faculty Fellowship. 
If $X$ is an infinite set, $\Sigma$ will denote the lattice of all topologies on $X$, $\Pi$ the lattice of principal topologies on $X, \Lambda$ the lattice of $T_{1}$ topologies on $X, \Lambda_{0}$ the lattice of $\boldsymbol{N}_{0}$-topologies on $X$ and $\Lambda_{c}$ the lattice of topologies such that every countable set is closed.

The author wishes to thank the referee for several suggestions which have helped to streamline the paper.

2. Mutually complementary topologies. Let us show that $|X|$ $\leqq w^{*}(\Lambda) \leqq 2^{|x|}$. Then it will be easy to suitably modify the construction to get the same result for $\Sigma, \Pi, \Lambda_{0}$ and $\Lambda_{c}$. We begin with a settheoretic result which says, intuitively, that if $Y$ is a set such that $|Y|=d \geqq \aleph_{0}$, then $Y$ can be split into halves $d$ ways such that if $x$ in $Y$ and a particular splitting of $Y$ are given, then there is a unique $z$ in $Y$ such that $x$ and $z$ are in the same half of the particular splitting of $Y$, but in different halves for every other splitting. The following notation will prove helpful. Suppose $Y$ is the union of nonempty pairwise disjoint sets $Y_{\alpha 0}$ and $Y_{\alpha 1}$. For each $x$ in $Y$, let $Y_{\alpha i(x)}$ be the "half" of $Y$ that contains $x$ and let $Y_{\alpha j(x)}$ be the other "half" of $Y$.

Lemma 1. Suppose $Y$ and $\Omega$ are sets, each of cardinal $d \geqq \boldsymbol{\aleph}_{0}$. For each $\alpha$ in $\Omega$, there exist nonempty pairwise disjoint sets $Y_{\alpha 0}$ and $Y_{\alpha 1}$ whose union is $Y$ such that if $\alpha$ in $\Omega$ and $x$ in $Y$ are given, then there is a $z$ in $Y$ such that

$$
Y_{\alpha i(x)} \cap\left(\cap\left\{Y_{\beta j(x)}: \beta \in \Omega-\{\alpha\}\right\}\right)=\{z\} .
$$

Proof. Let $W$ be a set with cardinal $d$ and suppose $F$ is the set of all functions from $W$ into $\{0,1\}$. Then $|F|=2^{d}$. There is a subset $S$ of $F$ that allows us to split $Y$ in the required ways.

Suppose $S_{1}=\left\{f \in F:\left|f^{-1}(\{1\})\right|\right.$ is finite $\}$, where we interpret zero as a finite cardinal, and $S_{0}$ is defined similarly with respect to 0 . If $S=S_{0} \cup S_{1}$, then since $S_{0}$ and $S_{1}$ may be considered as coordinatizing the finite subsets of $W$, it is clear that $|S|=d$. If $f \in S$ and $w \in W$, define $f_{w}$ to be that element of $F$ which agrees with $f$ at $w$ and disagrees with $f$ everywhere else. Certainly if $f \in S$ then $f_{w} \in S$.

Now, for each $w$ in $W$, split $S$ into "halves" by the definition

$$
S_{w i}=\{f \in S: f(w)=i\} ; \quad i=0,1 .
$$

Our choice of $S$ insures that none of these sets is empty. If $f$ in $S$ and $w$ in $W$ are given, then $f$ belongs to one and only one of $S_{w 0}$ and $S_{w 1}$. Furthermore, $f_{w}$ belongs to the same "w-half" of $S$ as $f$, but if $v \in W$ $-\{w\}$, then $f_{w}$ and $f$ are in different " $v$-halves". Moreover, it is clear that $f_{w}$ is the only element of $S$ with this property. Since there exist one-to-one mappings between $S$ and $Y$ and between $W$ and $\Omega$, the lemma follows. 
It is easy to see that this is the best possible result. For suppose $|\Omega|>d$ but the conclusion still holds. Pick $x$ in $Y$ and define the function $\phi_{x}: \Omega \rightarrow Y$ by letting $\phi_{x}(\alpha)$ be the unique $z$ in $Y$ that is the same " $\alpha$-half" of $Y$ as $x$, but in the other "half" of $Y$, for any $\beta$ in $\Omega-\{\alpha\}$. It is obvious that $\phi_{x}$ is one-to-one. This contradicts the supposition on the cardinality of $\Omega$.

Lemma 2. Suppose $X$ is an infinite set. Then $X$ carries a family of $|X|$ mutually $T_{1}$-complementary topologies. That is, $|X| \leqq w^{*}(\Lambda)$.

Proof. Express $X$ as the union of $|X|$ pairwise disjoint subsets, all with the same cardinal as $X$ and index these subsets by $Y$. Thus, $X=\cup\left\{X_{y}: y \in Y\right\}$. For each $y$ in $Y$, express $X_{y}$ as the union of $|X|$ pairwise disjoint subsets, all with the same cardinal as $X$ and index these sets by $\Omega$. Thus $X_{y}=\bigcup\left\{X_{y, \alpha}: \alpha \in \Omega\right\}$. In each $X_{y, \alpha}$, pick $|X|$ pairwise disjoint denumerable sets, $\left\{E_{y, \alpha, \beta}: \beta \in(\Omega-\{\alpha\})\right\} . \Omega$ can also be used to split $Y$, and therefore $X$ in to halves as in Lemma 1 .

Now, if $\gamma \in \Omega$, make the following definitions.

$$
\begin{aligned}
& A_{\gamma 0}=\bigcup\left\{E_{y, \gamma, \alpha}: y \in Y_{\gamma 0}, \alpha \in(\Omega-\{\gamma\})\right\}, \\
& A_{\gamma 1}=\bigcup\left\{E_{y, \gamma, \alpha}: y \in Y_{\gamma 1}, \alpha \in(\Omega-\{\gamma\})\right\}, \\
& B_{\gamma 0}=\bigcup\left\{X_{y}: y \in Y_{\gamma 0}\right\}, \\
& B_{\gamma 1}=\bigcup\left\{X_{y}: y \in Y_{\gamma 1}\right\}, \\
& E_{\gamma}=\bigcup\left\{E_{y, \alpha, \gamma}: y \in Y, \alpha \in(\Omega-\{\gamma\})\right\} .
\end{aligned}
$$

Then

(1) $B_{\gamma 0} \cup B_{\gamma 1}=X, B_{\gamma 0} \cap B_{\gamma 1}=\varnothing$,

(2) $A_{\gamma 0} \subset B_{\gamma 0}, A_{\gamma 1} \subset B_{\gamma 1}$,

(3) $\left(A_{\gamma 0} \cup A_{\gamma 1}\right) \cap E_{\gamma}=\varnothing$,

and if $\gamma \neq \rho$, then

(4) $A_{\gamma 0} \cap E_{\rho} \neq \varnothing, A_{\gamma 1} \cap E_{\rho} \neq \varnothing$,

(5) $\left(A_{\gamma 0} \cup A_{\gamma 1}\right) \cap\left(A_{\rho 0} \cup A_{\rho 1}\right)=\varnothing$,

(6) $E_{\gamma} \cap E_{\rho}=\varnothing$.

Suppose $x \in X$ but $x \notin E_{\gamma} \cup A_{\gamma 0} \cup A_{\gamma 1}$. There is a unique $y$ in $Y$ such that $x \in X_{v}$ and therefore by Lemma 1 , there is a unique $v$ in $Y$ associated with $y$ and $\gamma$. We make the definition

$$
C_{x \gamma}=\{x\} \cup\left(\cup\left\{E_{v, \alpha, \gamma}: \alpha \in(\Omega-\{\gamma\})\right\}\right) .
$$

Define $T_{\gamma}$ to be the topology generated by cofinite subsets of $B_{\gamma 0}$ and $B_{\gamma 1}$, singletons in $E_{\gamma}$ and

$$
\left\{C_{x \gamma}: x \notin E_{\gamma} \cup A_{\gamma 0} \cup A_{\gamma 1}\right\} .
$$

Since $B_{\gamma 0}$ and $B_{\gamma 1}$ are disjoint sets whose union is $X$, it is clear that $T_{\gamma}$ is a $T_{1}$ topology. Notice that every $T_{\gamma}$-open set intersects $E_{\gamma}$ and that every $T_{\gamma}$-open set containing a point of $A_{\gamma 0}\left(A_{\gamma 1}\right)$ is cofinite in $B_{\gamma 0}\left(B_{\gamma 1}\right)$. 
Lemma 2 will be verified if it can be shown that for any two distinct elements $\gamma$ and $\rho$ of $\Omega, T_{\gamma}$ and $T_{\rho}$ are $T_{1}$-complements. This means that $\sup \left\{T_{\gamma}, T_{\rho}\right\}$ must be discrete and $\inf \left\{T_{\gamma}, T_{\rho}\right\}$ must be the cofinite topology. If $x \in X$, then there is a unique $y$ such that $x \in X_{y}$ and $y \in Y_{\gamma i} \cap Y_{\rho j}, i, j=0,1$. First we show that $\{x\} \in$ $\sup \left\{T_{\gamma}, T_{\rho}\right\}$. If $x \in E_{\gamma} \cup E_{\rho}$, this is clear, since these sets are isolated in their respective topologies. If $x \in\left(A_{\gamma i}-E_{\rho}\right)$, then by (5), $x \notin E_{\rho} \cup A_{\rho 0}$ $\cup A_{\rho 1}$. Hence $C_{x_{\rho}} \cap B_{\gamma i}=\{x\}$. If $x \in\left(A_{\rho j}-E_{\gamma}\right)$, then by (5), $x \notin E_{\gamma}$ $\cup A_{\gamma 0} \cup A_{\gamma 1}$ and it follows that $C_{x \gamma} \cap B_{\rho j}=\{x\}$. Finally, if $x$ $\notin\left(E_{\gamma} \cup E_{\rho} \cup A_{\gamma i} \cup A_{\rho j}\right)$, then $C_{x \rho} \cap C_{x \gamma}=\{x\}$.

Suppose now that $U \in T_{\gamma} \cap T_{\rho}$ and $U \neq \varnothing$. We have noted previously that such a $U$ must intersect $E_{\gamma}$ and $E_{\rho}$. Pick $x$ such that $x \in U$ $\cap E_{\gamma}$. If $x \in A_{\rho i}$, then $U$ contains a cofinite subset of $B_{\rho i}$. Lemma $1 \mathrm{im}$ plies that each "half" of a splitting of $Y$ contains points in both "halves" of any other splitting. Thus, any cofinite subset of $B_{\rho i}$ must contain points in $A_{\gamma 0}$ and $A_{\gamma 1}$. Hence $U$ contains cofinite subsets of $B_{\gamma 0}$ and $B_{\gamma 1}$ and $U$ is cofinite in $X$. If $x \notin A_{\rho i}$, then by (6), $x \notin\left(E_{\rho}\right.$ $\cup A_{\rho 0} \cup A_{\rho 1}$ ), and $U$ must contain a cofinite subset of $C_{x \rho}$. But there is a $y$ such that $C_{x \rho}$ contains $E_{y, \gamma, \rho}$. Therefore, there is a $j, j=0,1$, such that $C_{x \rho} \cap A_{\gamma j}$ is infinite. This means that $U$ contains a cofinite subset of $B_{\gamma j}$ and hence contains points of $A_{\rho 0}$ and $A_{\rho 1}$ and must contain cofinite subsets of $B_{\rho 0}$ and $B_{\rho 1}$.

One more fact about the topologies defined in Lemma 2 will prove useful. Recall that Fréchet topologies are those topologies with the property that a point is in the closure of a set iff there is a sequence in the set converging to the point.

Lemma 3. The topologies defined in Lemma 2 are Fréchet topologies.

Proof. Consider the topology $T_{\gamma}$ as defined in Lemma 2. Suppose $G$ is an infinite subset of $X$ and $y \in \mathrm{Cl}(G)-G$. We wish to exhibit a sequence in $G$ that $T_{\gamma}$-converges to $y$.

Clearly $y \notin E_{\gamma}$. If $y \in A_{\gamma i}$, choose a sequence of distinct points in $G \cap B_{\gamma i}$. Any such sequence must converge to $y$ since every $T_{\gamma}$-open set containing $y$ must contain a cofinite subset of $B_{\gamma i}$. If $y \notin A_{\gamma 0} \cup A_{\gamma 1}$, choose a sequence of distinct points in $G \cap C_{y \gamma}$. Any such sequence must converge to $y$ since every $T_{\gamma}$-open set containing $y$ must contain a cofinite subset of $C_{\nu \gamma}$.

Theorem 1. Suppose $X$ is an infinite set and $L$ is one of the lattices $\Sigma, \Pi, \Lambda$ or $\Lambda_{0}$. Then $|X| \leqq w^{*}(L) \leqq 2^{|X|}$. If $X$ is uncountable, this inequality is also true for $\Lambda_{c}$. 
Proof. The upper bound on $w^{*}(L)$ is easily obtained since $X$ has $2^{|X|}$ subsets and the complementation conditions force each topology in a family of mutually complementary topologies to contain sets not in any other topology of the family. In the case of $\Pi$, this upper bound is no restriction at all, since $X$ has only $2^{|X|}$ principal topologies [8]. This is also true of $\Lambda_{0}$, if $X$ has cardinal at least $c$ [7].

Lemma 2 established the lower bound for $\Lambda$. A simple modification of the definition of $T_{\gamma}$ in Lemma 2 will give us the result for $\Sigma$. Merely change "cofinite subsets of $B_{\gamma 0}$ and $B_{\gamma 1}$ " to " $B_{\gamma 0}$ and $B_{\gamma 1}$ ". The argument of Lemma 2 now shows that if $\gamma \neq \rho$, then inf $\left\{T_{\gamma}, T_{\rho}\right\}$ is the indiscrete topology. Note also that the modified $T_{\gamma}$ 's clearly have the property that each point is contained in a minimal open set. Thus [9, Theorem 2.3] each modified $T_{\gamma}$ is a principal topology and the theorem is proved for $\Pi$. Theorem 1 of [7] states that a topology is an $\boldsymbol{\aleph}_{0}$-topology iff every nonclosed set contains a countably infinite subset with an accumulation point lying outside the set. Clearly every Fréchet $T_{1}$ topology is an $\boldsymbol{\aleph}_{0}$-topology. Therefore, Lemmas 2 and 3 show that $|X| \leqq w^{*}\left(\Lambda_{0}\right)$.

Finally, suppose $X$ is uncountable. Change the construction of Lemma 2 by choosing $\left\{E_{y, \alpha, \beta}: \beta \in(\Omega-\{\alpha\})\right\}$ to be a family of pairwise disjoint uncountable sets, modify the definition of $T_{\gamma}$ to read "cocountable subsets of $B_{\gamma 0}$ and $B_{\gamma 1}$ ", and the result follows for $\Lambda_{c}$. Clearly we can extend this technique to higher cardinals.

3. Complementation in $\Lambda_{0}$. It has been shown [7] that the lattice of $\boldsymbol{\aleph}_{0}$-topologies on an infinite set is not complemented. It is easy to extend a result of [2] to show that large classes of $\boldsymbol{\aleph}_{0}$-topologies have $\boldsymbol{\aleph}_{0}$-complements. Call a topological space $X$ splittable iff $X$ contains an infinite family of pairwise disjoint open sets. Call $X$ a $D N$-space iff each point in $X$ has at least one net in its complement that converges to it and whose range is a discrete subspace of $X$. If $N$ is the set of isolated points in $X$, call $(X-\mathrm{Cl} N)$ the open kernel of $X$. Now Theorem 1 of [2] states that if $(X, T)$ is a $T_{1}$-space whose open kernel is empty or a splittable $D N$-space, then $T$ has a $T_{1}$-complement $T^{\prime}$ that is compact on cofinite subsets of $X$.

It is easy to see, using an argument like that for Lemma 3, that $T^{\prime}$ is Fréchet. In addition, if $T$ is an $\boldsymbol{\aleph}_{0}$-topology, then $T$ and $T^{\prime}$ are $\boldsymbol{N}_{0^{-}}$ complements.

It has been noted that Fréchet $T_{1}$ topologies are $\boldsymbol{\aleph}_{\mathbf{0}}$-topologies.

REMARK 1. If $T$ is a Fréchet Hausdorff topology, a locally compact Hausdorff $\boldsymbol{\aleph}_{0}$-topology or a symmetrizable Hausdorff topology, then $T$ has an $\boldsymbol{\aleph}_{0}$-complement. 
Proof. Repeat the arguments of Corollaries 1, 2 and 3 of [2].

The ordinals through the first uncountable ordinal with the usual order topology yield a compact Hausdorff topology that is not an $\boldsymbol{\aleph}_{0}$-topology. Example 7.1 of [4] shows that a topology can be a compact Hausdorff $\boldsymbol{\aleph}_{0}$-topology and fail to be Fréchet. The example is the one-point compactification of the space $\Psi$ of Isbell (see [6, p. 79]).

4. An example. It may be of some interest to note that the methods of [2] also give one an easily pictured more elementary way of defining $\Sigma$-complements for nice topologies than the all inclusive methods of [9] or [12]. We illustrate the preceding statement (this is why Theorem 2 is stated for cardinal $c$ ) and at the same time prove

THEOREM 2. Every set of cardinal c carries a $T_{1}$ topology $T^{\prime}$ such that for any $T_{1}$ topology $S$ on a set $X$ of cardinal $c, T^{\prime}$ has a $T_{1}$-complement with a subspace homeomorphic to $(X, S)$. Analogous statements hold in $\Sigma$ and $\Lambda_{0}$.

Proof. Let $\left(R^{2}, T\right)$ be the plane and make the following definitions. For each integer $i$,

(a) $U_{i}=\{(x, y): i<x<i+1\} ; Y=U U_{i}$,

(b) $e_{i}=(i+1 / 2,0) ; E_{0}=\left\{e_{i}: i\right.$ is even $\}, E_{1}=\left\{e_{i}: i\right.$ is odd $\}$,

(c) $S_{i}=\{(i+1 / 2,1 / n): n \geqq 1\}$.

Also

(d) $A_{0}=\bigcup\left\{S_{i}: i\right.$ is even $\} ; A_{1}=\bigcup\left\{S_{i}: i\right.$ is odd $\}$,

(e) $B_{0}=A_{0} \cup\left[\left(Y-A_{1}\right) \cap\left(\cup\left\{U_{i}: i\right.\right.\right.$ is odd $\left.\left.\}\right)\right]$, $B_{1}=A_{1} \cup\left\{\left(Y-A_{0}\right) \cap\left(\cup\left\{U_{i}: i\right.\right.\right.$ is even $\left.\left.\}\right)\right]$,

(f) $D=Y-\left(A_{0} \cup A_{1} \cup E_{0} \cup E_{1}\right)=\left\{d_{\alpha}: \alpha \in \Omega\right\}$,

(g) $C_{\alpha}, \alpha \in \Omega$,

where $C_{\alpha}$ is defined as follows. If $d_{\alpha} \in D$, then there is an $i(\alpha)$ such that $d_{\alpha} \in U_{i(\alpha)}$. Now $i(\alpha)$ is either even or odd, and we can think of $U_{i(\alpha)}$ as being part of either the "even half" or "odd half" of $Y$. Let $C_{\alpha}$ be the union of $d_{\alpha}$ and all $e_{i}$ 's in the same half as $d_{\alpha}$, except $e_{i(\alpha)}$. Let $(T \mid Y)^{\prime}$ be the topology generated by

(i) $\{x\}, x \in E_{0} \cup E_{1}$,

(ii) $B_{i}, i=0,1$,

(iii) $C_{\alpha}, \alpha \in \Omega$,

(iv) $C_{Y}$, the cofinite topology on $Y$.

Then, as in Lemma 2 of [2], $(T \mid Y)^{\prime}$ is a $T_{1}$-complement of $T \mid Y$. Since $Y$ is an open dense subset of $\left(R^{2}, T\right)$, Theorem 5 of [11] assures us that if $T^{\prime}$ is the topology generated by

(v) $\{x\}, x \in R^{2}-Y$, 
(vi) $V \cup F, V \in(T \mid Y)^{\prime}$ and $F$ cofinite in $R^{2}-Y$, then $T^{\prime}$ is a $T_{1^{-}}\left(\boldsymbol{N}_{0^{-}}\right)$complement of $T$.

Notice that we can modify $T$ at points away from $E_{0} \cup E_{1}$ and the $S_{i}^{\prime}$ 's and still have $T^{\prime}$ for a $T_{\mathbf{1}^{-}}\left(\boldsymbol{N}_{0^{-}}\right)$complement. Specifically, suppose $S$ is a $T_{1^{-}}\left(\boldsymbol{N}_{0^{-}}\right)$topology on a set of cardinal $c$. Let $A$ be a closed circular disc entirely contained in the part of $U_{0}$ below the $x$-axis. Topologize $R^{2}$ with $T$-open sets in $R^{2}-A$ and a copy of $S$ in $A$. Clearly this topology and $T^{\prime}$ are $T_{1^{-}}\left(\boldsymbol{\aleph}_{0^{-}}\right)$complements. If we omit (iv) from the definition of $(T \mid Y)^{\prime}$ and modify (vi) to

(vii) $V \cup\left(R^{2}-Y\right), V \in(T \mid Y)^{\prime}$,

we see that a similar result holds for $\Sigma$.

It is clear from the construction that $T^{\prime}$ has $2^{2^{c}} T_{1}$-complements ( $2^{c} \boldsymbol{\aleph}_{0}$-complements, since $\left|\Lambda_{0}\right|=2^{c}$ if the base set has cardinal $c[\mathbf{7}$, Theorem 3]). It is known [8] that there are topologies in $\Sigma$ with $2^{2^{c}}$ $\Sigma$-complements.

Finally, notice that $T^{\prime}$ is a $T_{1}$-complement for much stronger topologies on $R^{2}$ than $T$, for example, the radial topology of [5].

\section{REFERENCES}

1. B. A. Anderson and D. G. Stewart, $T_{1}$-complements of $T_{1}$ topologies, Proc. Amer. Math. Soc. 23 (1969), 77-81. MR 39 \#6240.

2. B. A. Anderson, $A$ class of topologies with $T_{1}$-complements, Fund. Math. 69 (1970), 267-277.

3. G. Birkhoff, Lattice theory, 3rd ed., Amer. Math. Soc. Colloq. Publ., vol. 25, Amer. Math. Soc., Providence, R. I., 1967. MR 37 \#2638.

4. S. P. Franklin, Spaces in which sequences suffice. II, Fund. Math. 61 (1967), 5156. MR 36 \#5882.

5. S. P. Franklin and S. W. Williams, Nonregular separable spaces, Amer. Math. Monthly 75 (1968), 208.

6. L. Gillman and M. Jerison, Rings of continuous functions, Van Nostrand, Princeton, N. J., 1960. MR 22 \#6994.

7. R. E. Larson, A sublattice of the lattice of topologies, Notices Amer. Math. Soc. 17 (1970), 223. Abstract \#672-489.

8. P. S. Schnare, Infinite complementation in the lattice of topologies, Fund. Math. 64 (1969), 249-255. MR 39 \#3444.

9. A. K. Steiner, The lattice of topologies: structure and complementation, Trans. Amer. Math. Soc. 122 (1966), 379-398. MR 32 \#8303.

10. - Complementation in the lattice of $T_{1}$-topologies, Proc. Amer. Math. Soc. 17 (1966), 884-886. MR 33 \#1255.

11. A. K. Steiner and E. F. Steiner, Topologies with $T_{1}$-complements, Fund. Math. 61 (1967), 23-28. MR 37 \#5840.

12. A. C. M. van Rooij, The lattice of all topologies is complemented, Canad. J. Math. 20 (1968), 805-807. MR 37 \#3504.

Arizona State University, Tempe, Arizona 85281 